\title{
REVIEW
}

\section{Neurogenic bowel management after spinal cord injury: a systematic review of the evidence}

\author{
A Krassioukov ${ }^{1,2,3}$, JJ Eng 1,3,4,5, G Claxton ${ }^{3}$, BM Sakakibara ${ }^{3,5}$, S Shum ${ }^{3}$, and the SCIRE Research Team \\ ${ }^{1}$ International Collaboration on Repair Discoveries (ICORD), Vancouver, British Columbia, Canada; ${ }^{2}$ Division of Physical \\ Medicine and Rehabilitation, Department of Medicine, Vancouver, British Columbia, Canada; ${ }^{3}$ GF Strong Rehabilitation \\ Centre, Vancouver Coastal Health Care, Vancouver, British Columbia, Canada; ${ }^{4}$ Department of Physical Therapy, Vancouver, \\ British Columbia, Canada and ${ }^{5}$ Graduate Program in Rehabilitation Sciences, University of British Columbia, Vancouver, \\ British Columbia, Canada
}

\begin{abstract}
Study design: Randomized-controlled trials (RCTs), prospective cohort, case-control, pre-post studies, and case reports that assessed pharmacological and non-pharmacological intervention for the management of the neurogenic bowel after spinal cord injury $(\mathrm{SCl})$ were included.

Objective: To systematically review the evidence for the management of neurogenic bowel in individuals with $\mathrm{SCl}$.

Setting: Literature searches were conducted for relevant articles, as well as practice guidelines, using numerous electronic databases. Manual searches of retrieved articles from 1950 to July 2009 were also conducted to identify literature.

Methods: Two independent reviewers evaluated each study's quality, using Physiotherapy Evidence Database scale for RCTs and Downs and Black scale for all other studies. The results were tabulated and levels of evidence assigned.

Results: A total of 2956 studies were found as a result of the literature search. On review of the titles and abstracts, 57 studies met the inclusion criteria. Multifaceted programs are the first approach to neurogenic bowel and are supported by lower levels of evidence. Of the non-pharmacological (conservative and non-surgical) interventions, transanal irrigation is a promising treatment to reduce constipation and fecal incontinence. When conservative management is not effective, pharmacological interventions (for example prokinetic agents) are supported by strong evidence for the treatment of chronic constipation. When conservative and pharmacological treatments are not effective, surgical interventions may be considered and are supported by lower levels of evidence in reducing complications.
\end{abstract}

Conclusions: Often, more than one procedure is necessary to develop an effective bowel routine. Evidence is low for non-pharmacological approaches and high for pharmacological interventions. Spinal Cord (2010) 48, 718-733; doi:10.1038/sc.2010.14; published online 9 March 2010

Keywords: spinal cord injury; autonomic control; neurogenic bowel; management; evidence based; SCIRE

\section{Introduction}

Neurogenic bowel is a colonic dysfunction resulting from a lack of central nervous control (see Figure 1 for a schematic diagram of the gastrointestinal (GI) tract). It is a syndrome commonly observed in individuals with spinal cord injury (SCI). Lynch et al. ${ }^{1}$ assessed surveys from 467 persons with SCI and 668 age and gender-matched controls. They reported that quality of life was affected by fecal incontinence in $62 \%$ of SCI respondents compared with $8 \%$ of

Correspondence: Dr A Krassioukov, Department of Medicine, UBC/ICORD, ICORD, 818 West 10th Avenue, Vancouver, British Columbia, Canada V5Z 1M9. E-mail: krassioukov@icord.org

Received 17 September 2009; revised 14 January 2010; accepted 19 January 2010; published online 9 March 2010 controls. People with SCI suffered from greater extents of fecal urgency and required more time for bowel management. Coggrave et al. ${ }^{2}$ assessed surveys from over a thousand individuals over a year post-SCI and reported the presence of constipation (39\% of sample), hemorrhoids (36\%), and abdominal distension (31\%).

Neurogenic bowel dysfunction is a major physical and psychological problem for persons with SCI, as changes in bowel motility, sphincter control, coupled with impaired mobility and hand dexterity result to make bowel management a major life-limiting problem. As bowel dysfunction after SCI is a major source of morbidity, ${ }^{3,4}$ it is not surprising that improving bowel function alone or bladder/bowel functions is rated among the highest priorities among individuals with SCI. ${ }^{5,6}$ 


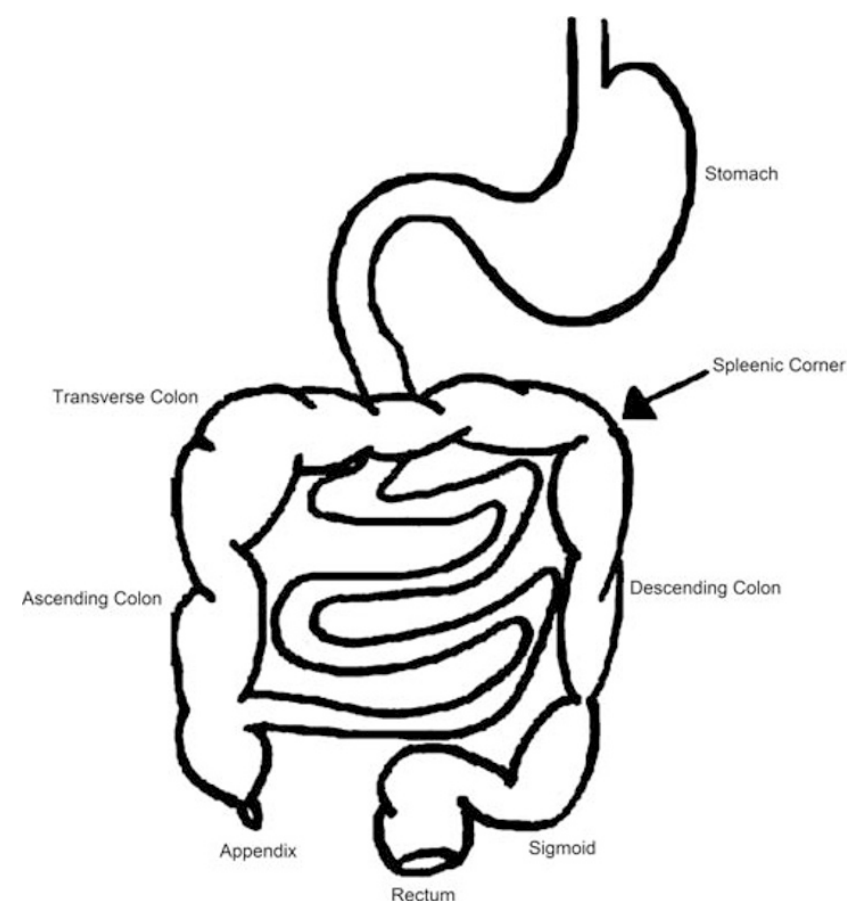

Figure 1 Schematic diagram of the $\mathrm{Gl}$ tract. Parasympathetic innervation is provided by the vagus (CNX) from the esophagus to the spleenic corner of the large intestine. Innervation of the Gl tract after the spleenic corner is provided by the sacral part of the parasympathetic nervous system (S2-S4). Sympathetic innervation to the upper GI tract is provided by the SPNs localized within the upper thoracic spinal segment (T1-T5); the small and a large intestine are controlled by SPNs localized within the T6-T12 spinal segments.

There are two distinct patterns in the clinical presentation of bowel dysfunction: injury above the conus medullaris results in upper motor neuron bowel syndrome and injury at the conus medullaris and cauda equine results in lower motor neuron bowel syndrome. ${ }^{7,8}$ The upper motor neuron bowel syndrome, or hyperreflexic bowel, is characterized by increased colonic wall and anal tones. Voluntary (cortical) control of the external anal sphincter is disrupted and the sphincter remains tight, thereby promoting retention of stool. The nerve connections between the spinal cord and the colon remain intact, and therefore, there is preserved reflex coordination and stool propulsion. The upper motor neuron bowel syndrome is typically associated with constipation and fecal retention at least in part because of external anal sphincter activity. ${ }^{7}$ Stool evacuation in these individuals occurs by means of reflex activity caused by a stimulus introduced into the rectum, such as an irritant suppository or digital stimulation. Lower motor neuron bowel syndrome, or areflexic bowel, is characterized by the loss of centrally mediated (spinal cord) peristalsis and slow stool propulsion. Lower motor neuron bowel syndrome is commonly associated with constipation and a significant risk of incontinence because of the atonic external anal sphincter and lack of control over the levator ani muscle that causes the lumen of the rectum to open. Completeness of injury also has a significant impact on bowel function in individuals with SCI. Those with an incomplete injury may retain the sensation of rectal fullness and ability to evacuate bowels, so no specific bowel program may be required; however, the pathophysiologic mechanisms of fecal incontinence and constipation in subjects with incomplete SCI are similar to subjects with complete SCI and preserved spinal sacral reflexes. ${ }^{9}$ Further, individual variations in bowel routing before SCI and preexisting conditions may also influence the pattern of bowel evacuation post-injury. Careful evaluation and individual approaches are, therefore, important for accurate diagnoses and prescription of treatments for bowel management after SCI.

Successful bowel management is multidimensional, and as such, treatments may be multifaceted, whereas others have focused on isolated strategies, such as dietary, pharmacological, electrical stimulation, and surgery. A systematic review of the evidence underlying bowel management has not been presented earlier. The Consortium for Spinal Cord Medicine $^{10}$ provided guidelines for neurogenic bowel based on evidence and best practice, although they are now over a decade old. The following systematic review was, therefore, undertaken to evaluate the evidence that supports the efficacy and effectiveness of the various strategies used to manage neurogenic bowel complications. These findings are part of the Spinal Cord Injury Rehabilitation Evidence project, ${ }^{11}$ available at www.scireproject.com.

\section{Materials and methods}

A key word literature search of articles, practice guidelines, and review articles was conducted to identify literature, published between 1950 and July 2009, evaluating treatments and therapies for neurogenic bowel in SCI populations. The key words of SCI, paraplegia, and tetraplegia were combined with neurogenic bowel, bowel management, incontinence, constipation, irregular, hemorrhoids, as well as treatment-specific terms, such as cisapride, colonic, colostomy, dietary fiber, laxative, and suppositories. Studies with SCI subjects as part of a mixed population sample were excluded if the results did not provide information specific to SCI.

All abstracts were reviewed by both a physiatrist and registered nurse with expertise in bowel and bladder management. For those studies deemed eligible for inclusion, their quality was then assessed by two trained independent reviewers, through the use of one of two assessment tools. Randomized-controlled trials (RCT) were evaluated with the Physiotherapy Evidence Database (PEDro). ${ }^{12}$ PEDro is a 10-point score, which assesses internal validity of a study, with higher scores indicating better methodological quality (9-10: excellent; 6-8: good; 4-5: fair; $<4$ : poor). ${ }^{13} \mathrm{~A}$ modified version of the Downs and Black evaluation tool was used to assess non-randomized studies. Scores on the modified Downs and Black tool range from 0 to 28 , with higher scores also indicating a higher-quality study. ${ }^{14}$ Conflicting assessments were resolved through discussion with a third reviewer. 
Levels of evidence developed by Sackett et al. ${ }^{15}$ were modified and collapsed into five categories, where Level $1=$ RCT with a PEDro score $\geqslant 6$; Level 2 = either a RCT with a PEDro score $\leqslant 5$, non-randomized prospective-controlled study, or cohort study; Level 3 =case-control study; Level 4 =either pre- and post-test or case series; and Level $5=$ either an observational report or case report involving a single subject or from clinical consensus. ${ }^{14}$

\section{Results}

As a result of the literature searches through the electronic databases, 2956 articles were found that met the search criteria. After eliminating duplicates and then reviewing the titles and abstracts, a total of 57 studies evaluating neurogenic bowel management strategies met the inclusion criteria. Management strategies evaluated in this review are either of non-pharmacological (conservative and non-surgical), pharmacological, or surgical in nature. Twenty-five studies assessed non-pharmacological conservative management strategies, including multifaceted programs (three studies), suppositories (five studies), dietary fiber (one study), reflex stimulation (one study), abdominal massage (one study), assistive devices (two studies), irrigation techniques (six studies), and functional electrical stimulation of skeletal muscles (six studies). Ten studies evaluated pharmacological treatment strategies, and 22 studies on surgical interventions, including implantation of electrical stimulation systems (nine studies), colostomy (nine studies), and the Malone procedure (four studies).

The following are the results for each type of management strategy.

Non-pharmacological (conservative and non-surgical)

Multifaceted programs. There are several factors that may influence bowel function, including diet, fluid consumption, and routine bowel evacuations. Multifaceted programs target more than one factor in an attempt to reduce colonic transit time as well as decrease the incidences of difficult evacuations. Improving the movement of stool through the GI tract is the most important part of any bowel management protocol after SCI. An array of interventions, as components of a bowel routine, are recommended for the management of neurogenic bowel after SCI. These include dietary recommendations, anorectal/perianal stimulation, timing the performance of the bowel routine with food intake (thus taking advantage of gastrocolonic and rectocolonic reflexes), and a variety of pharmacological agents. Unfortunately, only a limited number of studies evaluated the effects of different protocols on bowel function after SCI.

There is Level 4 evidence (from three pre-post studies; aggregate $N=65)^{16-18}$ that multifaceted bowel management programs reduce GI transit time, incidences of difficult evaluations, and duration of time required for bowel management (Table 1). Badiali et al.'s ${ }^{16}$ multifaceted bowel management program effectively reduced GI transit time, whereas Correa and Rotter's ${ }^{17}$ program reduced the incidence of difficult intestinal evacuation. Coggrave et al. ${ }^{18}$ recently modified the bowel management program originally proposed by Badiali et al. ${ }^{16}$ by including an additional step of manual evacuation and found a significant decrease in the number of bowel movement episodes requiring laxatives (from 62.8 to $23.1 \%$ ). These authors also reported a significant decrease in the mean duration of bowel management episodes with the introduction of this protocol. As all three studies incorporated several factors into the bowel management programs including diet, fluid consumption, and routine bowel practice, it is not possible to determine the key factor.

Use of suppositories. The use of chemical rectal agents (suppositories) is a common and often necessary component

Table 1 Multifaceted programs

\begin{tabular}{|c|c|c|}
\hline $\begin{array}{l}\text { Author; country; score; research design; total } \\
\text { sample size }\end{array}$ & Methods & Outcome \\
\hline $\begin{array}{l}\text { Coggrave et al. }{ }^{18} ; \text { United Kingdom; Downs } \\
\text { and Black score }=17 ; \text { pre-post; } N=17\end{array}$ & $\begin{array}{l}\text { Population: mean age: } 41.24 \text { years, range: } 19-59 \\
\text { years; level of injury: } 8 \text { cervical, } 8 \text { thoracic, and } \\
\text { conus medularis; motor complete. } \\
\text { Treatment: modified progressive bowel } \\
\text { management protocol designed by Badiali et al. }{ }^{16} \\
\text { OM: number of episodes requiring laxative and } \\
\text { duration of bowel management episodes. }\end{array}$ & $\begin{array}{l}\text { 1. Protocol increased successful bowel } \\
\text { management episodes without the use of } \\
\text { laxatives and decreased episodes requiring } \\
\text { laxatives. } \\
\text { 2. Duration of bowel management episodes } \\
\text { decreased as did the number of episodes } \\
\text { requiring manual evacuation. }\end{array}$ \\
\hline $\begin{array}{l}\text { Correa and Rotter }{ }^{17} ; \text { Chile; Downs and } \\
\text { Black score }=13 ; \text { pre-post; } N=38\end{array}$ & $\begin{array}{l}\text { Population: age range: } 19-71 \text { years; } 21 \text { complete, } \\
10 \text { incomplete; } 2 / 21 \text { tetraplegic and 19/21 } \\
\text { paraplegic. } \\
\text { Treatment: intestinal program administration. } \\
\text { OM: DIE scale, Gl symptoms. }\end{array}$ & $\begin{array}{l}\text { 1. DIE reduced from } 26.5 \text { to } 8.8 \% \text {, Gl symptoms, } \\
\text { and manual extraction reduced from } 53 \text { to } 37 \% \text {. }\end{array}$ \\
\hline $\begin{array}{l}\text { Badiali et al. }{ }^{16} \text {; Italy; Downs and Black } \\
\text { score }=13 ; \text { pre-post; } N=10\end{array}$ & $\begin{array}{l}\text { Population: mean age: } 33 \text { years, range: } 20-60 \\
\text { years; level of injury: C3-L4. } \\
\text { Treatment: modified diet, water intake, and } \\
\text { evacuation schedule. } \\
\text { OM: bowel movement frequency, CTT. }\end{array}$ & $\begin{array}{l}\text { 1. Bowel frequency increased at the end of } \\
\text { training. } \\
\text { 2. Reduction in gastrointestinal transit time. }\end{array}$ \\
\hline
\end{tabular}

Abbreviations: CTT, colonic transit time; DIE, difficult intestinal evacuation; GI, gastrointestinal; OM, outcome measures. 
Table 2 Suppositories

\begin{tabular}{|c|c|}
\hline $\begin{array}{l}\text { Author; country; score; research } \\
\text { design; total sample size }\end{array}$ & Methods \\
\hline $\begin{array}{l}\text { House and Stiens }{ }^{19} ; \text { USA; PEDro }=7 ; \\
\text { RCT; } N=15\end{array}$ & $\begin{array}{l}\text { Population: mean age: } 45 \text { years, range: } 26-61 \\
\text { years; level of injury: } 9 \text { cervical, } 6 \text { thoracic, } \\
11 \text { complete, } 4 \text { incomplete. } \\
\text { Treatment: } 10 \mathrm{mg} \text { HVB or } 10 \mathrm{mg} \text { PGB. } \\
\text { OM: time to flatus, flatus to stool flow, defecation } \\
\text { period. }\end{array}$ \\
\hline
\end{tabular}

Stiens et al. ${ }^{22}$; USA; Downs and Black score $=18$; non-randomized cross-over controlled; $N=14$

Frisbie $^{21}$; USA; Downs and Black score $=16$; prospective-controlled trial; $N=19$

Dunn and Galka ${ }^{20}$; USA; Downs and Black score $=12$; case series; $N=14$

Amir et al. ${ }^{23}$; USA; Downs and Black score $=9$; cohort; $N=7$
Population: mean age: 53.4 years; level of injury: C3-L1, 4 incomplete, 10 complete.

Treatment: PGB or HVB suppositories.

OM: time to flatus, flatus to stool flow, defecation period, clean up, total bowel care time.

Population: mean age: 64 years, range: 41-81 years; level of injury: 15 cervical, 4 thoracic (T1-7), 15 motor complete.

Treatment: PGB or HVB.

$O M$ : average time for complete bowel evacuation.

Population: age range: $27-67$ years; level of injury: C5-L1, 5 tetraplegics, 9 paraplegics.

Treatment: bisacodyl and Theravac SB

OM: bowel management time, bowel problems.

Population: age range: $21-76$ years; level of injury: C4-T12, 6 tetraplegics, 1 paraplegic.

Treatment: 1 week of therapy with one of the following: (1) two bisacodyl, (2) two glycerin,

(3) one mineral oil enema, or (4) one Theravac SB.

OM: total colonic and segmental CTT.
Outcome

1. PGB significantly less time than HVB.

2. No significant differences in flatus to stool flow.

3. PGB suppositories significantly decreased bowe care time.

1. Time to flatus: $H V B=31 \mathrm{~min}, P G B=12 \mathrm{~min}$; defecation period: $\mathrm{HVB}=58 \mathrm{~min}, \mathrm{PGB}=32 \mathrm{~min}$; total bowel care time: $\mathrm{HVB}=102 \mathrm{~min}$ $\mathrm{PBG}=51.2 \mathrm{~min}$

2. Digital stimulations required for the bowel care sessions: $\mathrm{HVB}=5.0, \mathrm{PGB}=3.2$.

1. All patients experienced a shortening of bowel care time with $\mathrm{PGB}$. Average time for bowel evacuation was $2.4 \mathrm{~h}$ with $\mathrm{HVB}, 1.1 \mathrm{~h}$ with $\mathrm{PGB}$.

1. Mean evacuation times were lower with the use of Theravac SB than with bisacodyl.

1. Total CTT significantly reduced with Theravac SB, no significant difference between Theravac SB and mineral oil enema, but both had significantly shorter CTT than bisacodyl or glycerin.

2. Theravac SB had the shortest CTT and was best for symptom reduction.

Abbreviations: CTT, colonic transit time; HVB, hydrogenated vegetable-oil base; OM, outcome measures; PBG, polyethylene glycol base; PEDro, Physiotherapy Evidence Database; RCT, randomized-controlled trial.

Table 3 Dietary fiber

\begin{tabular}{|c|c|c|}
\hline $\begin{array}{l}\text { Author; country; score; research } \\
\text { design; total sample size }\end{array}$ & Methods & Outcome \\
\hline $\begin{array}{l}\text { Cameron et } \text { al. }^{24} ; \text { Australia; } \\
\text { Downs and Black score }=10 \text {; } \\
\text { case series; } N=11\end{array}$ & $\begin{array}{l}\text { Population: age range: } 19-53 \text { years; level of injury: C4-T12; } 1 \\
\text { incomplete and } 10 \text { complete; } 7 \text { tetraplegics and } 4 \text { paraplegics. } \\
\text { Treatment: increased fiber intake ( } 40 \mathrm{~g} \text { Kellogg's All Bran). } \\
\text { OM: stool weight, CT, and segmental transit time, bowel } \\
\text { evacuation time and fiber intake. }\end{array}$ & $\begin{array}{l}\text { 1. Fiber intake increased from } 25 \text { to } 31 \mathrm{~g} \text { per day. } \\
\text { 2. Mean CTT increased from } 28.2 \text { to } 42.2 \mathrm{~h} \text {. } \\
\text { 3. Rectosigmoid CTT increased from } 7.9 \text { to } \\
23.3 \mathrm{~h} \text {. } \\
\text { 4. No change in stool weight and evacuation } \\
\text { time. }\end{array}$ \\
\hline
\end{tabular}

Abbreviations: CTT, colonic transit time; OM, outcome measures.

of a successful bowel management program. Bisacodyl (dulcolax) and glycerin are the most common active ingredients in these suppositories. Five studies (aggregate $N=69)^{19-23}$ examined the effect of suppositories on bowel management in SCI including one RCT and two controlled trials, which were not randomized (Table 2). There is Level 1 evidence (from one good-quality RCT, however, with few subjects), ${ }^{19}$ in addition to lower levels of evidence, ${ }^{20-22}$ to support polyethylene glycol-based suppositories for bowel management. These suppositories resulted in a clinically significant decrease in the amount of nursing time for persons requiring assistance and less time to perform bowel care for the independent individual. The total bowel care time with the polyethylene glycol-based suppository is significantly less compared with hydrogenated vegetable oil-based bisacodyl suppositories. ${ }^{21-23}$

Dietary fiber. There is Level 4 evidence (from one case series; $N=11)^{24}$ that high fiber diets may cause colonic transit time to increase, rather than decrease (Table 3). The results of the one case series suggest that increasing dietary fiber in SCI patients does not have the same effect on bowel function as has been shown earlier in individuals with normalfunctioning bowels. The effect may actually be the opposite of the desired result. ${ }^{24}$ Therefore, adding more fiber alone does not improve bowel function. 
Reflex stimulation of the GI tract. Digital rectal stimulation is often used as an adjunct to laxatives and enemas to facilitate bowel evacuation. There is Level 4 evidence (from one pre-post study; $N=6$ ) (Table 4) that digital rectal stimulation increases motility in the left colon by activating preserved anorectal colonic reflexes. ${ }^{25}$

Abdominal massage. There is Level 4 evidence (from one pre-post study; $N=24$ ) that abdominal massage significantly shortened total colonic transit time, reduced abdominal distension, and increased frequency of bowel movements per week (Table 5). ${ }^{26}$ Ayas et al. ${ }^{26}$ first established baseline values with 24 subjects who participated in a 3-week standard bowel program in which they received a standard diet containing 15-20 g of fiber per day and underwent daily digital stimulation. Patients then received at least $15 \mathrm{~min}$ of daily abdominal massage for a minimum of 15 days. The massage began at the cecum and extended along to the length of the colon to the rectum.

Assistive devices. Assistive devices have been evaluated as means to improve bowel evacuation in individuals with SCI. These include a standing table and a modified toilet seat (two studies, aggregate $N=21$ ) (Table 6). There is Level 5 evidence (from one case report with one subject) that a standing table alleviates constipation in individuals with SCI. ${ }^{27}$ Hoenig et al. $^{27}$ reported the case of an individual with SCI who, through the use of a standing table, doubled the frequency of his bowel movements and reduced time spent on bowel care. There is Level 4 evidence (from one post-test study) that a washing toilet seat with visual feedback can reduce time spent on bowel care. ${ }^{28}$ Uchikawa et al. ${ }^{28}$ developed a new procedure to induce bowel movements using a toilet set equipped with an electronic bidet that provides water flow to

Table 4 Reflex stimulation

\begin{tabular}{|c|c|c|}
\hline $\begin{array}{l}\text { Author; country; score; research } \\
\text { design; total sample size }\end{array}$ & Methods & Outcome \\
\hline $\begin{array}{l}\text { Korsten et al. }{ }^{25} ; \text { USA; Downs and } \\
\text { Black score }=12 \text {; pre-post; } N=6\end{array}$ & $\begin{array}{l}\text { Population: mean age: } 50.2 \text { years, range: } 44-50 \text { years; } \\
\text { level of injury: C5-T10; } 4 \text { paraplegics, } 2 \text { tetraplegics. } \\
\text { Treatment: DRS. } \\
\text { OM: colorectal monometry: mean number of peristaltic } \\
\text { waves per minute; amplitude of contractions; colonic } \\
\text { motility. }\end{array}$ & $\begin{array}{l}\text { 1. Increase in peristaltic waves per minute during } \\
\text { DRS }(1.9 \pm 0.5 \text { per minute) and after DRS } \\
(1.5 \pm 0.3 \text { per minute) average amplitude was } \\
43.4 \pm 2.2 \mathrm{~mm} \mathrm{Hg} \text { (range: } 0.7-250) \text {. } \\
\text { 2. Peristaltic contractions in the left colon were } \\
\text { accompanied by increased motility. }\end{array}$ \\
\hline
\end{tabular}

Abbreviations: DRS, digital rectal stimulation; OM, outcome measures.

Table 5 Abdominal massage

\begin{tabular}{lll}
\hline $\begin{array}{l}\text { Author; country; score research design } \\
\text { total sample size }\end{array}$ & Methods & Outcome \\
\hline Ayas et al. ${ }^{26}$; Turkey; Downs and & Population: mean age: 39.8 years, range: $33.1-46.6$ years; & 1. Mean frequencies of defecation increased from \\
Black score $=18 ;$ pre-post; $N=24$ & level of injury: C4-L3; 15 complete; 9 incomplete. & $3.79 \pm 2.15$ to $4.61 \pm 2.17$ per week. \\
& Treatment: abdominal massage from the cecum to the & 2. CTT decreased from $90.60 \pm 32.67$ to \\
& rectum. & $72 \pm 34.10 \mathrm{~h}$. \\
& OM: CTT, frequency of defecation. & \\
\hline
\end{tabular}

Abbreviations: CTT, colonic transit time; OM, outcome measures.

Table 6 Assistive devices

\begin{tabular}{|c|c|c|}
\hline $\begin{array}{l}\text { Author; country; score; research } \\
\text { design; total sample size }\end{array}$ & Methods & Outcome \\
\hline $\begin{array}{l}\text { Hoenig et al. }{ }^{27} ; \text { USA; Downs and } \\
\text { Black score }=15 \text {; case report; } N=1\end{array}$ & $\begin{array}{l}\text { Population: } 62 \text {-year-old male with T12-L1, paraplegia. } \\
\text { Treatment: standing table, } 5 \text { times per week. } \\
\text { OM: frequency of bowel movements and length of } \\
\text { bowel care episodes. }\end{array}$ & $\begin{array}{l}\text { 1. Bowel movements increased from } 10 \text { to } 18 \text {. } \\
\text { 2. The time spent on bowel care reduced from } \\
21 \text { to } 13 \mathrm{~min} \text {. }\end{array}$ \\
\hline $\begin{array}{l}\text { Uchikawa et al. }{ }^{28} ; \text { Japan; Downs and } \\
\text { Black score }=13 ; \text { post-test; } N=20\end{array}$ & $\begin{array}{l}\text { Population: mean age: } 46.3 \text { years, range: } 18-73 \text { years; } \\
\text { level of injury: } 11 \text { cervical, } 7 \text { thoracic, } 2 \text { lumbar. } \\
\text { Treatment: toilet seat equipped with an electronic } \\
\text { bidet, a light, and camera monitor. } \\
\text { OM: time for bowel movement, residual stool. }\end{array}$ & $\begin{array}{l}\text { 1. } 75 \% \text { (15 subjects) of participants decrease time } \\
\text { of bowel routine to }<30 \text { min compare with } \\
\text { only } 35 \% \text { ( } 7 \text { subjects) with usual bowel care. }\end{array}$ \\
\hline
\end{tabular}

Abbreviation: OM, outcome measures. 
the anorectal area. A camera and light are included to facilitate location of the anorectal area.

Irrigation techniques. Six studies (aggregate $N=445$ ) evaluated irrigation techniques to improve bowel management (Table 7). ${ }^{29-34}$ There is Level 4 evidence (from one case-series study evaluating 31 persons with SCI) that supports using pulsed water irrigation (intermittent rapid pulses) to remove stool in individuals with SCI. ${ }^{29}$ There is also Level 1 evidence (from one large good-quality multisite RCT with 87 subjects), ${ }^{30}$ Level 4 evidence (from two pre-post study evaluating 55 and 32 persons with SCI), ${ }^{31,32}$ and Level 5 evidence (from an observational study) ${ }^{33}$ that support the use of the transanal irrigation systems. In the RCT, the Peristeen Anal
Irrigation system (Coloplast A/S, Kokkedal, Denmark) showed reduced frequency of lower urinary traction, improved fecal continence, and reduced constipation after 10 weeks of use when compared with the conservative bowel treatment (Paralyzed Veterans of America Clinical Practice Guidelines for Bowel Management). ${ }^{30}$ Positive responses were greatest in the more severely impaired participants who used a wheelchair or were confined to bed (versus ambulatory participants). Findings were similar after 10 and 3 weeks of use in the two pre-post studies. ${ }^{31,32}$ In the Del Popolo et al..$^{32}$ study, 9 out of 32 study subjects also either reduced or eliminated their use of pharmaceuticals. In the observational study, Faaborg et al. ${ }^{33}$ found 98 of the 211 patients had successful outcomes after a mean follow-up of 19 months, and 74 patients were successfully using irrigation

Table 7 Irrigation techniques

\begin{tabular}{ll}
\hline $\begin{array}{l}\text { Author; country; score; research } \\
\text { design; total sample size }\end{array}$ & Outcome \\
\hline
\end{tabular}

Christensen et al. $^{30}$; Denmark; PEDro score $=7$; randomizedcontrolled trial; $N=87$

Christensen et al. $^{31}$; USA; Downs and Black score $=20$; pre-post; $N=55$

Christensen et al. ${ }^{34}$; Denmark; Downs and Black score $=17$; retrospective interviews and case series; $N=29 ; 19$ $\mathrm{SCl}$ patients

Del Popolo et al. ${ }^{32}$; Italy; Downs and Black score $=14$; pre-post; $N=32$

Faaborg et al. ${ }^{33}$; Denmark; Downs and Black score $=13$; observational; $N=211$
Population: TAl group: mean age: 47.5 years; level of injury: T10-S1, 23 complete and 12 incomplete. Conservative management group: mean age: 50.6 years; T10-S1, 23 complete and 22 incomplete. Treatment: TAI (peristeen anal irrigation system) or conservative management (PVA clinical guidelines) for 10 weeks.

OM: CCCSS, FIGS, fecal incontinence score.

Population: mean age $47.5 \pm 15.5$ years; level of injury: 61 supraconal, 37 complete, 25 incomplete Treatment: TAI (peristeen anal irrigation) for 10 weeks.

OM: CCCSS; FIGS; and NBD.

Population: ECC group: mean age: 39.9 years, range: 7-72 years; level of injury: T2-T11, conal or cauda equina injuries $(n=15)$. MACE group: mean age: 32.8 years, range: $15-66$ years; level of injury: C5-T2 $(n=4)$. Treatment: ECC versus MACE.

$O M$ : colorectal function, practical procedure, impact on daily living and QoL, general satisfaction.

Population: median age: 31.6 years, 13 complete, 14 incomplete

Treatment: TAI (peristeen anal irrigation) for a 3 weeks.

OM: QoL; use of pharmaceuticals, incidence of incontinence and constipation, abdominal pain or discomfort.

Population: median age 49 years, range: 7-81 years; etiology: 74 traumatic, 32 spinal bifida, 29 prolapsed intervertebral disk, 38 other, 38 non-SCI.

Treatment: TAl

OM: rate of success (treatment was successful if (1) currently using TAl, (2) the patient used TAl until death, or (3) symptoms resolved while using TAI).

Population: age: NA; level of injury: 8 tetraplegic, 4 complete; 23 paraplegic, 9 complete. Treatment: pulsed irrigation

OM: efficacy of technique, outpatient use.
1. TAl group scored better on symptom-related quality-of-life tool, CCCSS, FIGS, and NBD.

2. Improvement found in the TAl group was not confined to the more physically able patients.

3. The frequency of urinary tract infection was lower in the TAl group.

1. CCCSS, FIGS, and NBD scores improved.

2. TAl significantly reduced constipation, improved anal continence, and improved symptom-related QoL.

1. The ECC was successful in $53 \%$ of participants (8 subjects).

2. The MACE procedure was successful in $75 \%$ of participants (3 subjects).

3. Successful treatment with the ECC or the MACE led to significant improvements in QoL.

1. Significant increase in QoL scores and improvement of constipation.

2. Significant decrease in abdominal pain and incidence of incontinence.

3. Nine patients reduced or eliminated pharmaceutical use.

1. 42 patients stopped TAl in the first 3 months.

2. Success in 98 patients after 19 months; and 73 patients after 3 years of follow-up.

3. Abdominal pain, minor rectal bleeding, and general discomfort were observed in 101 patients.

1. Success in removing stool in all, but three patients.

2. Eleven patients had multiple procedures.
Puet et al. ${ }^{29} ;$ USA; Down
and Black score $=12 ;$ case series; $N=31$
Clinic Constipation Scoring System; ECC, enema co

Abbreviations: CCCSS, Cleveland Clinic Constipation Scoring System; ECC, enema continence catheter; FIGS, St Mark's Fecal Incontinence Grading System; MACE, malone antegrade continence enema; NBD, neurogenic bowel dysfunction; OM, outcome measures; PEDro, Physiotherapy Evidence Database; PVA, Paralyzed Veterans of America; QoL, quality of life; TAl, transanal irrigation. 


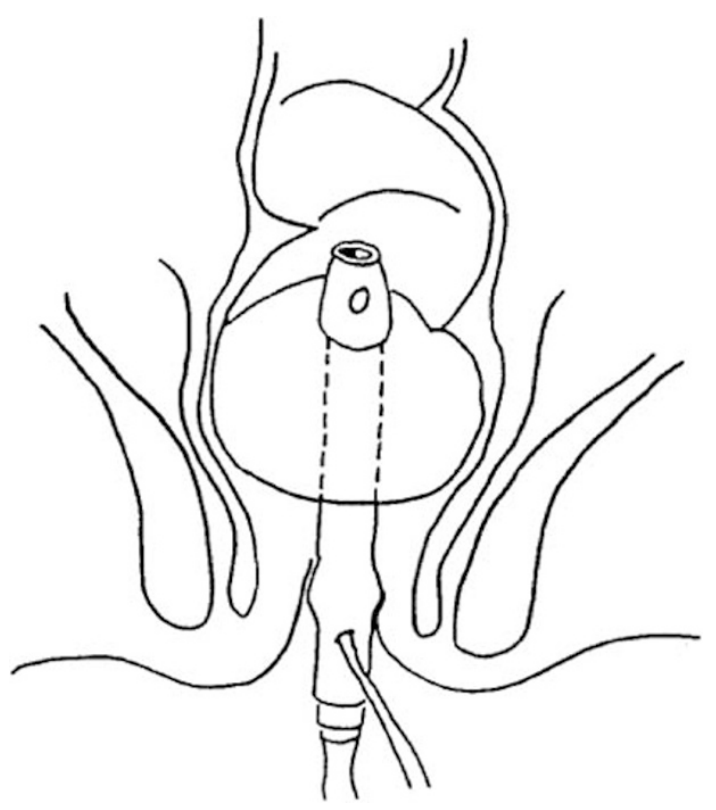

Figure 2 Schematic diagram of the enema continence catheter. A catheter is inserted into the rectum and a balloon is inflated to hold the catheter in place during the administration of an enema. After installing the enema, the balloon is deflated, the catheter is removed, and the bowel content will empty. Reprinted with permission from Macmillan Publishers Ltd: Spinal Cord, reference ${ }^{34}$ copyright (2000).

techniques after 3 years. In this study, successful outcomes were defined as irrigation still being used during follow-up, patients who used irrigation until they died, and patients whose symptoms had resolved while using irrigation techniques. Finally, there is Level 4 evidence (from one retrospective review) that the Enema Continence Catheter can be used to treat the neurogenic bowel with improved fecal continence and improved quality of life $^{34}$ (see Figure 2 for a diagram of the ECC).

Functional electrical and magnetic stimulation of skeletal muscles. Six studies (aggregate $N=74$ ) evaluated electrical or magnetic stimulation on skeletal muscles as a modality to improve colonic transit time in SCI with one study being an RCT (Table 8). ${ }^{35-40}$

There is Level 1 evidence (from one good-quality RCT, however, with few subjects) that external electrical stimulation of the abdominal wall muscles can improve bowel management for individuals with tetraplegia. ${ }^{35}$ They used an overnight abdominal belt with embedded electrodes to provide the stimulus. Level 2 evidence also exists (from a prospective-controlled trial), in which $25 \mathrm{~min}$ of electrical simulation of the abdominal muscles per day, 5 days a week, for 8 weeks, resulted in accelerated colonic transit times when compared with the placebo-control group. ${ }^{36}$ There is supporting lower evidence as Lin et al. ${ }^{37,38}$ showed that the use of external abdominal functional magnetic stimulation reduced colonic transit time in individuals with SCI.

There is Level 4 evidence (from one pre-post study with two subjects) that posterior tibial nerve stimulation improves bowel management for those with incomplete SCI. ${ }^{39}$ Although preliminary results for posterior tibial nerve stimulation seem promising, it is important to note that the statistical significance of the improvements in clinical and physiological parameters were not reported and the study involved only two subjects. ${ }^{39}$ Level 4 evidence also exists for the use of functional magnetic stimulation on the thorax and lumbosacral nerves (simulation placed at T9 and L3 spinal processes) to reduce colonic transit times and selfreported symptoms of constipation. ${ }^{40}$

\section{Pharmacological agents}

Ten studies (aggregate $N=199$ ) evaluated the effect of treatment strategies using pharmacology to enhance bowel management (Table 9). ${ }^{41-50}$ These studies addressed the chronic constipation after SCI and used agents to promote transit through the GI tract. Of all the bowel management literature, studies involving pharmacological agents had the highest quality with 6 of the 10 studies being small, but good-quality RCTs (PEDro $\geqslant 6$ ).

There is Level 1 evidence (from two RCTs) ${ }^{41,42}$ and Level 2 evidence (from one RCT) ${ }^{43}$ that cisapride significantly reduces colonic transit time for chronic constipation. There is also Level 1 evidence (from one good-quality RCT) ${ }^{44}$ that prucalopride increases stool frequency, improves stool consistency, and decreases GI transit time. Prucalopride is a novel, highly selective serotonin receptor agonist with enterokinetic properties that facilitate cholinergic and excitatory non-adrenergic, non-cholinergic neurotransmission. ${ }^{44}$ There is Level 2 evidence (from one controlled trial, which was not randomized; $N=20)^{45}$ that intravenous administration of metoclopramide corrects impairments in gastric emptying. Metoclopramide is a potent dopamine receptor antagonist with prokinetic properties and Segal et al. $^{45}$ found that impaired gastric emptying is correlated with decreased drug absorption. There is Level 1 evidence (from two good-quality RCTs) ${ }^{46,47}$ in support of the use of neostigmine (a reversible cholinesterase inhibitor) or the combination of neostigmine and glycopyrrolate. When administered intravenously, Korsten et al. $^{46}$ found both neostigmine and the combination of neostigmine/glysopyrrolate to improve stool expulsion over normal saline. Similarly, Rosman et al. ${ }^{47}$ found the combination to reduce total bowel evacuation time over placebo injections. There is Level 1 evidence (from one good-quality RCT with 71 participants) that fampridine (selective potassium channel blocker) can increase the number of days with bowel movements in approximately one-fifth of the subjects. ${ }^{48}$

\section{Surgical treatments}

Implantation of electrical stimulation systems. Nine studies (aggregate $N=137$ ) evaluated the effects of surgical implantation of electrical stimulation systems (Table 10). ${ }^{51-59}$ There is Level 2 evidence (from one prospective, non-randomizedcontrolled trial) ${ }^{51}$ that supports the use of sacral anterior root stimulation to reduce severe constipation in complete injuries. Binnie et al. ${ }^{51}$ had found that an implanted Brindley stimulator did not reduce oro-cecal time for individuals with 
Table 8 Functional electrical and magnetic stimulation of skeletal muscles

\begin{tabular}{ll}
\hline $\begin{array}{l}\text { Author; country; score; research } \\
\text { design; total sample size }\end{array}$ & Methods \\
\hline Korsten et $a .^{35} ;$ USA; & Population: mean age: 48 years, range: $34-62$ years; \\
PEDro $=6 ; \mathrm{RCT} ; N=8$ & 6 tetraplegics, 2 paraplegics. \\
& Treatment: abdominal belt with embedded electrodes, \\
& used for 6 bowel care sessions over 2 weeks. Subjects \\
& did not know whether the device was activated or not. \\
& OM: time to first stool, time for total bowel care.
\end{tabular}

Hascakova-Bartova et al. $^{36}$; Belgium; Downs and Black score $=21$; prospectivecontrolled trial; $N=10$

Lin et al. ${ }^{37}$; USA; Downs and Black score $=12$; pre-post; $N=15$

Lin et al. ${ }^{38}$; USA; Downs and Black score $=11$; pre-post; $N=9$

Tsai et al. ${ }^{40}$; Taiwan; Downs and Black score $=19$; pre-post; $N=22$

Mentes et al. $^{39}$; Turkey; Downs and Black score $=13$; pre-post; $N=2$
Population: mean age: 42 years, range: 23-61 years; level of injury C3-T10.

Treatment: abdominal NMES, administered for 25 min per day, 5 days a week, for 8 weeks. OM: EMG; FVC; CTT

Population: mean age: NA; level of injury: C3-L1. Treatment: Protocol 1: FMS on the transabdominal and lumbosacral regions. Protocol 2: 5-week stimulation period.

OM: rectal pressure and total and segmental transit times.

Population: mean age: 42 years; level of injury: C3-C7 $(n=4)$ and 5 able-bodied controls. Treatment: FMS along T9 spinous process. OM: rate of gastric emptying.

Population: mean age: 46.7 years, range: $22-65$ years. Treatment: FMS of the thorax and lumbosacral nerves, in 20-min sessions twice daily for 3 weeks. OM: CTT; Knowles-Eccersley-Scott symptom questionnaire.

Population: 51-year-old woman (discectomy for lumbar disc herniation) and a 31-year-old man (10-year history of lumbar cavernous hemangioma). Treatment: 30 min of tibial nerve stimulation every other day for 4 weeks, then repeated every 2 months for 3 times.

OM: physiologic, clinical and QoL parameters.
Outcome

1. Activation of the abdominal belt significantly reduced the time to first stool and time for total bowel care.

2. Time to first stool and time for total bowel care was significantly shortened in 6 subjects with tetraplegia, but not in the 2 subjects with paraplegia.

1. NMES decreased FVC in the treatment group, but not in the control group.

2. NMES accelerated CTT in the ascending, transverse, and descending colon.

1. Rectal pressures increased with sacrolumbar stimulation, and with transabdominal stimulation.

2. The mean CTT decreased from 105.2 to $89.4 \mathrm{~h}$ after 5 week of stimulation.

1. Gastric emptying half/time of post-stimulation was significantly shorter in $\mathrm{SCl}$ subjects than the baseline ( $84 \pm 11$ versus $59 \pm 13 \mathrm{~min}$ ).

2. There was also a significant improvement in the percentage of gastric emptying with FES at 20, 60,90 , and $120 \mathrm{~min}$ in compression at baseline.

1. Mean CTT decreased from 62.6 to $50.4 \mathrm{~h}$

2. Mean scores on the Knowles-Eccersley-Scott symptom questionnaire decreased from 24.5 to 19.2 points.

1. Patients showed improvements in Wexner FI score, FIQL score, clinical parameters, and physiological measurements. Significance of improvements not reported in this study.

Abbreviations: CTT, colonic transit times; EMG, electromyography; FIQL, fecal incontinence quality of life scales; FMS, functional magnetic stimulation; FVC, forced vital capacity; NA, information not available; NMES, neuromuscular electrical stimulation; OM, outcome measures; QoL, quality of life; RCT, randomizedcontrolled trial.

SCI. However, subjects in the stimulator group did experience a significant increase in defecation compared with the SCI group.

Furthermore, recent pre-post studies using sacral nerve root stimulation yielded improvements in bowel function, including the ability to evacuate spontaneously, ${ }^{52}$ reduced bowel program times, ${ }^{53,59}$ elimination of autonomic dysreflexia related to bowel management ${ }^{53}$ increased quality of life, ${ }^{53,57}$ elimination of manual help for defecation, ${ }^{54}$ and reduced number of incontinence episodes. ${ }^{57,58}$ Conflicting evidence exists, however, on the effects of resting and squeeze canal pressures. ${ }^{57,58}$ Level 4 evidence also exists in support of sacral nerve stimulation in the treatment of fecal incontinence in patients suffering from cauda equine syndrome. ${ }^{55}$ Gstaltner et al. ${ }^{55}$ found an improved fecal continence, quality of life, and deliberate retention of feces in all subjects.
There is Level 4 evidence (from one pre-post study with results from only one subject presented) that the Praxis FES system for skeletal muscle stimulation paired with extradural electrodes for bowel and bladder stimulation increases the frequency of defecation and decreases time required for bowel care. ${ }^{56}$

Colostomy. A colostomy is the surgical formation of an artificial anus by connecting the colon to an opening in the abdominal wall. SCI patients who receive elective colostomy usually have exhausted all other medical treatments available to them for bowel management. Nine studies (aggregate $N=590$ ) examined the effect of colostomy after SCI (Table 11). ${ }^{60-68}$ Given the ethical nature of this treatment (that is few safe options once they reach the point of 


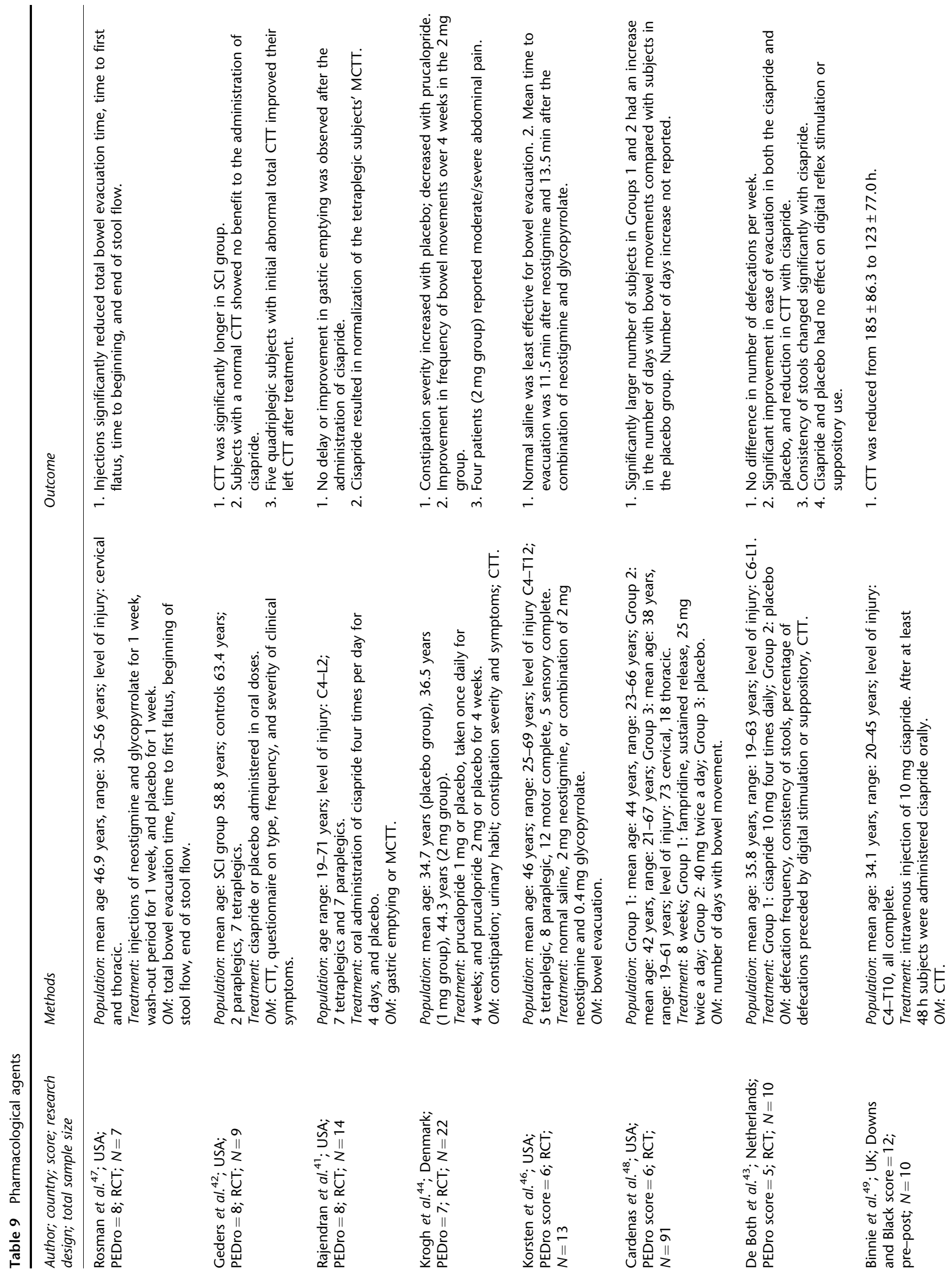


requiring a colostomy), most studies are pre-post or retrospective in nature. There is Level 4 evidence (from five studies), ${ }^{60-64}$ and Level 5 evidence (from one study) ${ }^{68}$ that colostomy reduces the number of hours spent on bowel care. There is Level 4 evidence (from one retrospective pre-post study ${ }^{60}$ that colostomy greatly simplifies bowel care routines. There is also Level 4 evidence (from one case study) ${ }^{63}$ that colostomy reduces the number of hospitalizations caused by GI problems and improves physical health, psychosocial adjustment, and self-efficacy areas within quality of life. Similarly, Levels 4 and 5 evidence exists (from a post-study and an observational study) that colostomy improves the independence, and thus quality of life, of SCI patients. ${ }^{67,68}$

Malone antegrade continence enema and the enema continence catheter. The malone antegrade continence enema (MACE) is an approach using a surgically created entry into the large intestine to irrigate the intestine (see Figure 3). The procedure involves connecting the appendix to the abdominal wall and making a valve mechanism that allows catheterization of the appendix, but avoids leakage of stool through it, thus forming an appendicostomy. ${ }^{69}$ Consequently, a catheter can be introduced to the patient through the stoma and an enema administered. ${ }^{34}$ Owing to the wash-out effect and perhaps the stimulated colonic peristaltic, the colon and rectum will empty, thus preventing fecal incontinence and constipation. ${ }^{34}$ Four case series (aggregate $N=122$ ) examined the effect of MACE on bowel function (Table 12). ${ }^{34,70-72}$ There is Level 4 evidence (from four case series) that MACE successfully treats the neurogenic bowel and patients reported improvements, including improved fecal continence, less time for bowel evacuation, reduced autonomic dysreflexia, and improved quality of life. $^{34,70-72}$

\section{Discussion}

The management of bowel disorders and, in particular, the constipation that is so common in SCI patients has remained essentially unchanged for several decades. This systematic review, however, has revealed some new areas of promise, including new assistive devices, such as irrigation techniques and electrical stimulation.

This review identified 57 studies on neurogenic bowel management strategies. A multifaceted approach is generally the first conservative approach to neurogenic bowel management, which generally includes evacuation schedules, diet, and fluid intake recommendations, as well as digital evaluation. As some form of multifaceted approach is now standard of care, a trial, which does not include some diet/ fluid recommendations, is not ethical. From the results of the three pre-post studies on multifaceted bowel management, it is apparent that the protocols are highly individualized, and, although there is generally a benefit, the results can be varied among participants.

Coggrave et $a .^{2}$ reported that digital evacuation was the most common intervention (reported by $56 \%$ of the 1334 


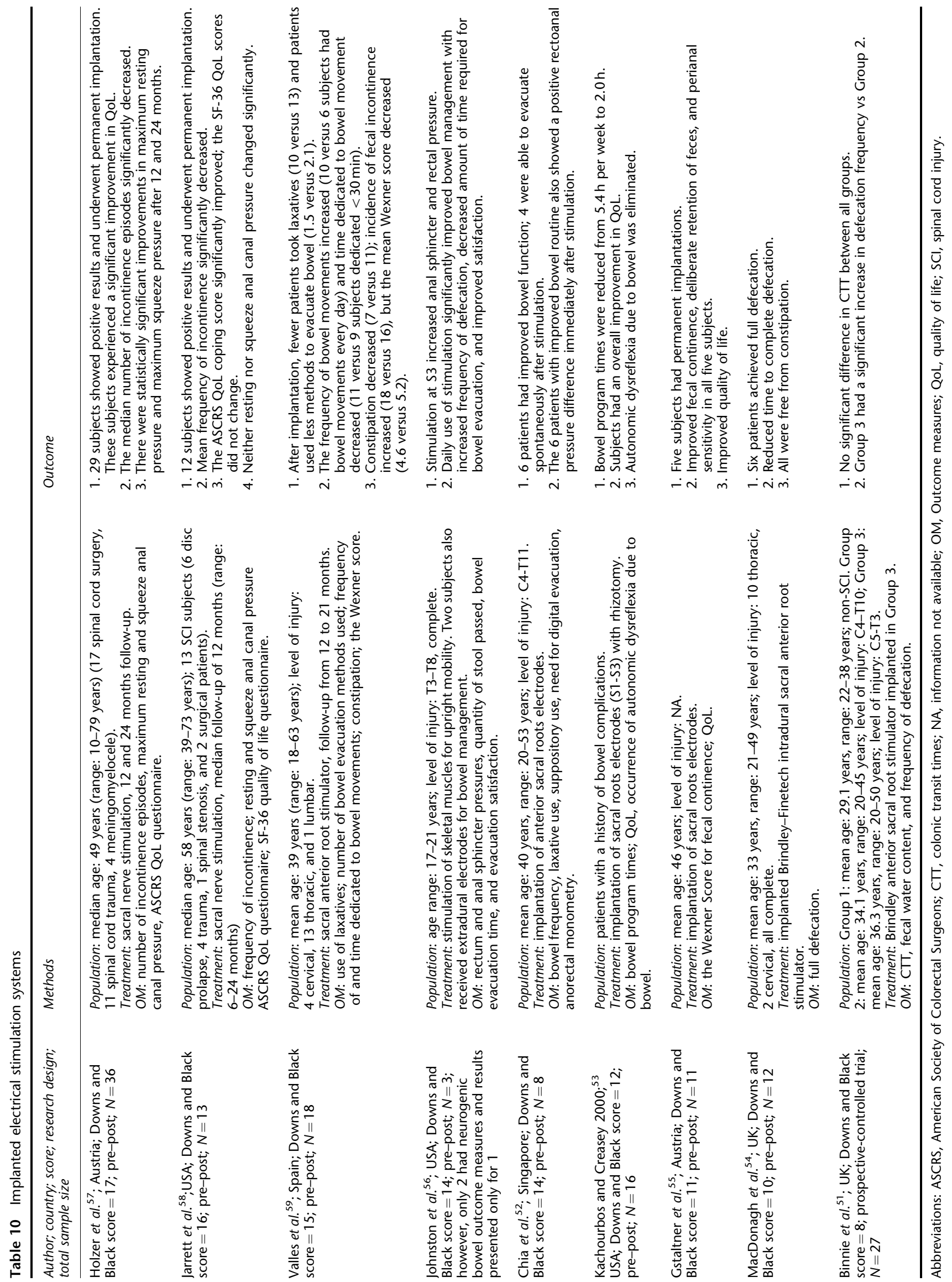


Table 11 Colostomy

$\begin{array}{lll}\text { Author; country; score; research } & \text { Methods } & \text { Outcome } \\ \text { design; total sample size } & & \text { Ont }\end{array}$

Randell et al. ${ }^{65}$; New Zealand; Downs and Black score $=17$; post-test; $N=52$

Kelly et al. ${ }^{62}$; UK; Downs and Black score $=15$; case series; $N=14$

Munck et al. ${ }^{67}$; Belgium; Downs and Black score $=13$; observational; $N=23$

Luther et al. ${ }^{66}$; USA; Downs and Black score $=12$; post-test; $N=370$

Safadi et al. ${ }^{68}$; USA; Downs and Black score $=12$; post-test; $N=45$

Branagan et al. ${ }^{64}$; UK; Downs and Black score $=11$; case series; $N=32$

Stone et al. ${ }^{61}$; USA; Downs and Black score $=11$; case series; $N=7$

Frisbie et al. ${ }^{60}$; USA; Downs and Black score $=9$; post-test; $N=20$

Rosito et al. ${ }^{63}$; USA; Downs and Black score $=8$; case series; $N=27$
Population: age range: 22-87 years; level of injury: 10 cervical, 16 lumbar/lower thoracic. Treatment: 26 subjects with colostomy, 26 subjects without colostomy. OM: QoL: systemic symptoms, emotional, social, work, and bowel function.

Population: mean age at time of operation: 54.8 years, range: $20-65$ years; level of injury: C4-T11; 3 cervical, 10 thoracic, 1 lumbar. Treatment: left iliac fossa colostomy $(n=12)$ and right iliac fossa ileostomy $(n=2)$. OM: time spent on bowel care per week; independence in bowel care; QoL.

Population: age range at stoma formation: 22-72 years; level of injury: cervical to lumbar. Treatment: intestinal stoma formation. OM: bowel care, QoL.

Population: case (colostomy): age range 20-89 years; Controls (regular bowel routine): age range: $20-89$ years; level of injury: NA. Treatment: colostomy OM: bowel care-related items; QoL.

Population: mean age: 55.9 years; 21 tetraplegic, 24 paraplegic.

Treatment: right and left colostomy and ileostomy. OM: QoL, CTT, bowel care time.

Population: mean age at injury: 28.9 years; level of injury: 10 cervical, 18 thoracic, 3 lumbar. Treatment: colostomy.

OM: bowel care; results of surgery.

Population: Mean age: 51.6 years; level of injury: C4-T10.

Treatment: Colostomy

OM: bowel care time.

Population: median age: 55 years, range: 27-75 years; level of injury: 9 cervical, 11 thoracic. Treatment: colostomies or ileostomies. OM: bowel care time, bowel care frequency, bowel care-related complaints.

Population: mean age: 62.9 years; level of injury: C4-L3, 17 complete and 10 incomplete injuries. Intervention: colostomy OM: QoL questionnaire.
1. No significant difference between the 2 groups in general well being, emotional, social, or work functioning.

1. Colostomy patients: mean time spent on bowel care per week decreased; independence with bowel care increased; quality of life improved in 10 patients

2. lleostomy patients: no change in mean time spent on bowel care per week.

1. Average time spent on bowel care per week decreased from 5.95 to $1.5 \mathrm{~h}$.

2. 3/10 reported cutaneous irritations and 1 reported detachment of the pocket.

3. 9/10 had easier bowel care, and 6 had greater independence.

1. Mean responses to the QoL items were high, but a large number of respondents in both groups expressed dissatisfaction with bowel care.

2. No difference between groups in bowel care outcomes or bowel-related QoL items.

1. СТT was significantly longer in the right colostomy.

2. In all surgeries, QoL increased and bowel care time decreased.

1. Time spent on bowel care per week decreased from 10.3 to $1.9 \mathrm{~h}$.

2. 18 patients experienced greater independence. 25 patients wished they had been offered a stoma earlier. No patients wanted a stoma reversal.

1. Time spent on bowel care decreased dramatically.

1. Bowel care frequency increased, bowel care duration lowered.

2. Except for foul odor, bowel care complaints decreased post-operation.

1. QoL significantly improved.

2. Lowered number of hospitalizations by $70.4 \%$.

3. Time spent on bowel care was reduced.

Abbreviations: NA, information not available; OM, Outcome measures; QoL, quality of life.

participants with SCI). One study ${ }^{25}$ showed that digital rectal stimulation increases peristaltic waves in the left colon, thus increasing motility in this segment. Gastrocolonic and anorectal reflexes can be successfully incorporated into a bowel routine for individuals with SCI. It is well known that after breakfast, a gastric distention can activate bowel motility and morning defecation. ${ }^{73,74}$ Furthermore, digital anorectal stimulation has been shown to be useful in bowel evacuation after $\mathrm{SCI}^{75}$ and is potentially useful in bowel management after SCI. At least, in part, an anorectal colonic reflex that results in enhanced contractions of the descending colon and rectum may contribute to bowel evacuation in individuals with SCI. Stimulation through abdominal massage seems to have some positive outcomes on colonic function, thus controlled trials are feasible for this intervention and need to be performed. 


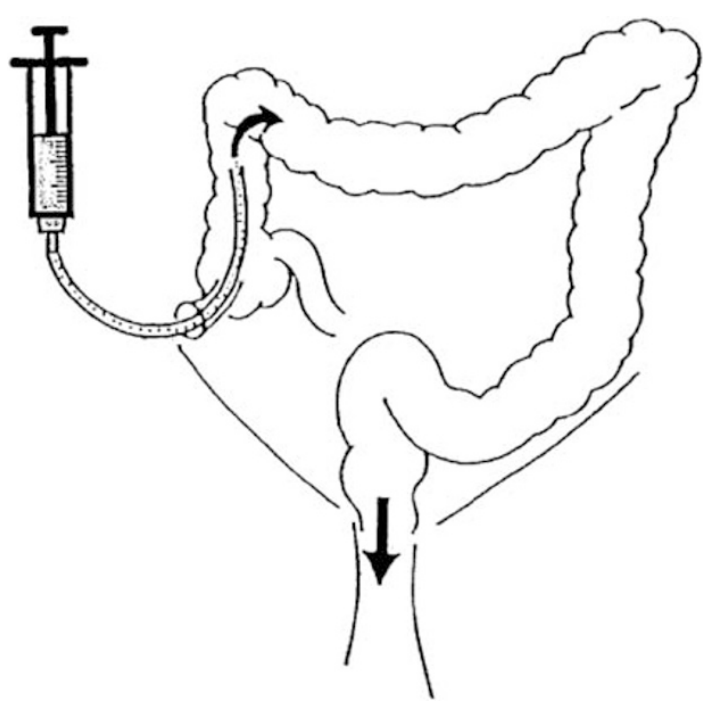

Figure 3 Diagram of the malone anterograde continence enema (MACE). The MACE procedure involves a surgical operation to bring out the appendix through the skin, thereby forming an appendicostomy. An enema may be introduced through the abdominal wall stoma. The enema produces a wash-out effect and stimulates colon peristalsis, which then evacuates the contents in the colon. Reprinted with permission from Macmillan Publishers Ltd: Spinal Cord, reference ${ }^{34}$ copyright (2000).
Conservative treatment may also include diet, although the study by Cameron et al. ${ }^{24}$ sends a cautionary message that dietary fiber may have different effects in people with SCI compared with able-bodied persons. Adding more fiber alone does not improve bowel function; however, more research is needed to assess its effectiveness.

Clinical experience shows that despite their best efforts, some persons with SCI are unable to achieve an effective, regular bowel routine and thus, other methods may be explored. The one large, multisite RCT showed that transanal irrigation reduces time spent on bowel management, dependency on others for help, and the frequency of defecation-related symptoms (that is abdominal pain, anorectal pain, nausea). ${ }^{30}$ In addition, transanal irrigation seems to alleviate fecal incontinence and constipation more so than conservative bowel management. ${ }^{30}$ Pulse water irrigation is another promising technique and consists of supplying intermittent, rapid pulses of warm water into the rectum to break up stool impactions and to stimulate peristalsis. ${ }^{29}$ Pulsed irrigation evacuation is a safe and effective method for individuals with SCI who develop impactions or do not have an effective bowel routine. ${ }^{29}$

Electrical or magnetic stimulation devices can be expensive, and are not readily accessible to patients. These devices

Table 12 The malone antegrade continence enema

\begin{tabular}{|c|c|}
\hline $\begin{array}{l}\text { Authorr; country; score; research } \\
\text { design; total sample size }\end{array}$ & Methods \\
\hline
\end{tabular}

Christensen et al. ${ }^{34}$; Denmark; Downs and Black score $=17$; retrospective interviews and case series; $N=29 ; 19 \mathrm{SCl}$ patients
Population: ECC group: mean age: 39.9 years, range: 7-72 years; level of injury: T2-T11, conal or cauda equina injuries $(n=15)$. MACE group: mean age: 32.8 years, range: $15-66$ years; level of injury: C5-T2 $(n=4)$. Treatment: ECC versus MACE. OM: colorectal function, practical procedure, impact on daily living, and QoL, general satisfaction.

Population: mean age: 36 years, range: 29-47 years; level of injury: T5 complete, C6 complete, C7 incomplete.

Treatment: MACE.

OM: bowel incontinence; subjective patient satisfaction.

Population: mean age: 51 years (range: 17-84 years), various $\mathrm{SCl}$ etiologies.

Treatment: ACE or ACE combined with colostomy, mean follow-up $=75$ months

OM: a 44-item questionnaire, including use of the ACE; functional results and side effects; overall satisfaction with bowel function and quality of life; success of treatment.

Population: mean age: 32.5 years, range: 22-47 years; level of injury: C6 complete, C7 incomplete, T5 complete, C6.

Treatment: MACE.

OM: number of fecal incontinence episodes per week; time for evacuation.
1. The ECC was successful in $53 \%$ of participants (8 subjects).

2. The MACE procedure was successful in $75 \%$ of participants (3 subjects).

3. Successful treatment with the ECC or the MACE led to significant improvements in QoL.

1. $2 \mathrm{SCl}$ subjects experienced fecal incontinence before the operation. Post-operative, both became continent.

2. All SCl subjects were satisfied with their outcomes and rated their QoL higher.

3. All subjects reduced toileting times.

1. ACE success rate was $74 \%$ (51 of 69 subjects) in reducing constipation and incontinence.

2. Complications occurred in 30 patients, including would infection, urinary tract infection, stenosis of the appendicostomy, and problems with catheterization.

3. Both social function and QoL significantly improved.

1. $3 \mathrm{SCl}$ subjects experienced fecal incontinence before the operation. Post-operative, all became continent.

2. All subjects were able to evacuate within $30 \mathrm{~min}$. case series; $N=7$; $4 \mathrm{SCl}$ patients

Abbreviations: ACE, antegrade colonic enema; ECC, enema continence catheter; MACE, malone antegrade continence enema; OM, outcome measures; SCl, spinal cord injury. 
generally have weak evidence. The exception was the stimulation, which used an abdominal belt with embedded electrodes and resulted in reduced bowel time. ${ }^{35}$

Often, medication is considered a later resort (although before surgery), with its use reserved for persons with severe constipation and where modification of the conservative bowel program has failed. Prokinetic agents are presumed to promote transit through the GI tract, thereby decreasing the length of time needed for stool to pass through the intestines and increasing the amount of stool available for evacuation. Pharmacological studies were high-quality (good-quality) RCTs, with positive results for cisapride, prucalopride, metoclopramide, neostigmine (administered both with and without glycopyrrolate), and fampridine; however, both cisapride and prucalopride are not currently available in either Canada or the USA because of adverse side effects. Chemical rectal agents (suppositories) are used commonly by persons with SCI to maintain or enhance a successful bowel management program. The glycerin suppository is a mild local stimulus and lubricating agent. Bisacodyl (dulcolax) is an irritant that acts directly on the colonic mucosa producing peristalsis throughout the colon. The most commonly used laxative suppositories contain $10 \mathrm{mg}$ of bisacodyl powder distributed within a hydrogenated vegetable-oil base. ${ }^{19}$ However, polyethylene glycol-based suppositories seem to be more effective than those in hydrogenated vegetable oil-based bisacodyl supporities. ${ }^{20-22}$

As conservative or pharmacological management of neurogenic bowel dysfunction is successful in $67 \%$ of the SCI population, ${ }^{76}$ surgical interventions provide an option for those with severe chronic constipation or when conservative management fails. Surgical interventions, such as implantation of electrodes, colostomies, and the MACE procedure, are options to treat chronic severe constipation when conservative management is ineffective. Although no studies have determined the best time to reevaluate the effectiveness of conservative management strategies, 1 year post-injury is likely a good time to determine whether surgical interventions will provide better outcomes. ${ }^{76}$ Han et al $^{77}$ report that $93 \%$ of individuals have a stable bowel management status 12 months post-SCI, and similarly, Lynch et al. ${ }^{1}$ report that after 12 months, bowel function does not change significantly with time or age.

The outcomes from implanted electrical stimulation techniques (implantation of epineural electrodes for skeletal muscle activation, and implantation of epidural or anterior sacral root electrodes) require consideration, as the participants in these trials are most often individuals who do not respond well to conservative management and/or have a history of bowel complications. ${ }^{40}$ Thus, any improvements from this select group might be viewed with encouragement, given their lack of response to other treatments. Owing to the invasiveness of these surgical procedures, control groups were not included in the implanted stimulator studies. However, in the future studies, investigators should be encouraged to randomize participants once implanted with the electrodes into a period of 'on-stimulation' versus 'offstimulation.' Such a design would permit interpretation of potential placebo effects of the surgery and instrumentation.
The need for colostomies and the MACE are often viewed as a failure of rehabilitation services. However, it is of importance to note that colostomy is a safe, effective method of managing severe and chronic GI problems, and perianal pressure ulcers in persons with SCI. Our systematic review shows that colostomies reduce the number of hours spent on bowel care, ${ }^{60-64}$ reduce the number of hospitalizations caused by GI problems ${ }^{63}$ and bowel care-related complaints, ${ }^{60}$ simplify bowel care routine, ${ }^{60}$ and improve quality of life. ${ }^{62,63,67}$ In addition, many patients wished to have the colostomy performed earlier. ${ }^{64}$ As described by Safadi et al., ${ }^{68}$ the left colostomy may be preferred because it preserves colic surfaces to absorb water and prevent dehydration; thus, feces are less liquid and discharges less frequently than with right colostomies. Using a decision analysis to examine the optimal treatment for chronic refractory constipation in SCI, Furlan et al. ${ }^{76}$ found that the MACE procedure had the best long-term outcomes (reduced complication rates, lower incidence of autonomic dysreflexia, and fitted with patient preferences). As more data becomes available, however, results could change on further critical evaluation of the impact of surgical interventions on the patient's well being.

\section{Conclusion}

Multifaceted bowel management programs are the first approach to neurogenic bowel programs and are supported by lower-level evidence (pre-post studies). Often, more than one procedure is necessary for individuals that are unable to develop an effective bowel routine. Digital rectal stimulation is often incorporated within these multifaceted programs and increases motility in the left colon in individuals with SCI. Diet and fluid intake are important components of multifaceted bowel management programs, although there is a need for further research to examine the optimal level of dietary intake in spinal cord injured patients. Transanal irrigation is a promising technique to reduce constipation and fecal incontinence. When conservative management is not effective, prokinetic agents, such as cisapride, prucalopride, metoclopramide, neostigmine, and fampridine, are supported by strong evidence for the treatment of chronic constipation in persons with SCI; however, more research is required on the safety of several of the agents before their regular use. Surgical interventions, such as colostomy, MACE, and implanted stimulators, are not routinely used, although all are supported by lower levels of evidence (prepost studies) in reducing bowel-related complications and improving quality of life. Overall, more intervention trials are needed to assess management programs for neurogenic bowel among individuals with SCI, especially trials involving multiple centers. Ideally, the use of common and validated scoring systems, such as the Neurogenic Bowel Dysfunctions score, and those found in the International Bowel Function data sets will be implemented such that comparison of results and meta-analyses may be conducted to further our knowledge on the treatment and management of neurogenic bowel after SCI. 


\section{Conflict of interest}

The authors declare no conflict of interest.

\section{Acknowledgements}

We acknowledge the support we received from the SCI Solutions Network and the Ontario Neurotrauma Foundation. JJE is supported by Career Scientist awards from the Michael Smith Foundation for Health Research and the Canadian Institutes of Health Research (CIHR MSH-63617). BMS is supported by a CIHR graduate scholarship.

\section{References}

1 Lynch AC, Antony A, Dobbs BR, Frizelle FA. Bowel dysfunction following spinal cord injury. Spinal Cord 2001; 39: 193-203.

2 Coggrave $M$, Norton C, Wilson-Barnett J. Management of neurogenic bowel dysfunction in the community after spinal cord injury: a postal survey in the United Kingdom. Spinal Cord 2009; 47: 323-330.

3 Stone JM, Nino-Marcia M, Wolfe VA, Perkash I. Chronic gastrointestinal problems in spinal cord injury patients: a prospective analysis. Am J Gastroenterol 1990a; 85: 1114-1119.

4 Han TR, Kim JH, Kwon BS. Chronic gastrointestinal problems and bowel dysfunction in patients with spinal cord injury. Spinal Cord 1998; 36: 485-490.

5 Glickman S, Kamm MA. Bowel dysfunction in spinal-cord-injury patients. Lancet 1996; 347: 1651-1653.

6 Anderson KD. Targeting recovery: priorities of the spinal cordinjured population. J Neurotrauma 2004; 21: 1371-1383.

7 Stiens SA, Bergman SB, Goetz LL. Neurogenic bowel dysfunction after spinal cord injury: clinical evaluation and rehabilitative management. Arch Phys Med Rehabil 1997; 78: S86-S102.

8 Singal AK, Rosman AS, Bauman WA, Korsten MA. Recent concepts in the management of bowel probles after spinal cord injury. $A d v$ Med Sci 2006; 51: 15-22.

9 Valles M, Mearin F. Pathophysiology of bowel dysfunction in patients with motor incomplete spinal cord injury: comparison with patients with motor complete spinal cord injury. Dis Colon Rectum 2009; 52: 1589-1597.

10 Consortium for Spinal Cord Medicine. Neurogenic Bowel Management in Adults with Spinal Cord Injury. In Clinical Practice Guidelines. Paralyzed Veterans of America, Washington, DC, 1998.

11 SCIRE Team. SCIRE: Spinal Cord Injury Rehabilitation Evidence 2006; Available at: http://www.icord.org/scire/.

12 Moseley AM, Herbert RD, Sherrington C, Maher CG. Evidence for physiotherapy practice: a survey of the Physiotherapy Evidence Database (PEDro). Aust J Physiother 2002; 48: 43-49.

13 Foley NC, Teasell RW, Bhogal SK, Speechley MR. Stroke rehabilitation evidence-based review: methodology. Top Stroke Rehabil 2003; 10: 1-7.

14 Eng JJ, Teasell RW, Miller WC, Wolfe DL, Townson AF, Aubut JA et al. Spinal cord injury rehabilitation evidence: method of the SCIRE systematic review. Top Spinal Cord Inj Rehabil 2007; 13: $1-10$.

15 Sackett DL, Strauss SE, Richardson WS, Rosenberg W, Haynes RB. Evidence-Based Medicine: How to Practice and Teach EBM. Churchill Livingstone: Edinburgh, 2000.

16 Badiali D, Bracci F, Castellano V, Corazziari E, Fuoco U, Habib FI et al. Sequential treatment of chronic constipation in paraplegic subjects. Spinal Cord 1997; 35: 116-120.

17 Correa GI, Rotter KP. Clinical evaluation and management of neurogenic bowel after spinal cord injury. Spinal Cord 2000; 38: 301-308.

18 Coggrave M, Burrows D, Durand MA. Progressive protocol in the bowel management of spinal cord injuries. Br J Nurs 2006; 15: $1108-1113$.
19 House JG, Stiens SA. Pharmacologically initiated defecation for persons with spinal cord injury: effectiveness of three agents. Arch Phys Med Rehabil 1997; 78: 1062-1065.

20 Dunn KL, Galka ML. A comparison of the effectiveness of Therevac SB and bisacodyl suppositories in SCI patients' bowel programs. Rehabil Nurs 1994; 19: 334-338.

21 Frisbie JH. Improved bowel care with a polyethylene glycol based bisacadyl suppository. J Spinal Cord Med 1997; 20: 227-229.

22 Stiens SA, Luttrel W, Binard JE. Polyethylene glycol versus vegetable oil based bisacodyl suppositories to initiate side-lying bowel care: a clinical trial in persons with spinal cord injury. Spinal Cord 1998; 36: 777-781.

23 Amir I, Sharma R, Bauman WA, Korsten MA. Bowel care for individuals with spinal cord injury: comparison of four approaches. J Spinal Cord Med 1998; 21: 21-24.

24 Cameron KJ, Nyulasi IB, Collier GR, Brown DJ. Assessment of the effect of increased dietary fibre intake on bowel function in patients with spinal cord injury. Spinal Cord 1996; 34: 277-283.

25 Korsten M, Singal AK, Monga A, Chaparala G, Khan AM, Palmon $\mathrm{R}$ et al. Anorectal stimulation auses increased colonic motor activity in subjects with spinal cord injury. I Spinal Cord Med 2007; 30: 31-35.

26 Ayas S, Leblebici B, Sozay S, Bayramoglu M, Niron EA. The effect of abdominal massage on bowel function in patients with spinal cord injury. Am J Phys Med Rehabil 2006; 85: 951-955.

27 Hoenig H, Murphy T, Galbraith J, Zolkewitz M. Case study to evaluate a standing table for managing constipation. Sci Nurs 2001; 18: 74-77.

28 Uchikawa K, Takahashi H, Deguchi G, Liu M. A washing toilet seat with a CCD camera monitor to stimulate bowel movement in patients with spinal cord injury. Am J Phys Med Rehabil 2007; 86: 200-204.

29 Puet TA, Jackson H, Amy S. Use of pulsed irrigation evacuation in the management of the neuropathic bowel. Spinal Cord 1997; 35: 694-699.

30 Christensen P, Bazzocchi G, Coggrave M, Abel R, Hultling C, Krogh $\mathrm{K}$ et al. A randomized, controlled trial of transanal irrigation versus conservative bowel management in spinal cordinjured patients. Gastroenterology 2006; 131: 738-747.

31 Christensen P, Bazzocchi G, Coggrave M, Abel R, Hulting C, Krogh $\mathrm{K}$ et al. Outcome of transanal irrigation for bowel dysfunction in patients with spinal cord injury. J Spinal Cord Med 2008; 31: 560-567.

32 Del Popolo G, Mosiello G, Pilati C, Lamartina M, Battaglino F, Buffa $\mathrm{P}$ et al. Treatment of neurogenic bowel dysfunction using transanal irrigation: a multicenter Italian study. Spinal Cord 2008; 46: $517-522$.

33 Faaborg PM, Christensen P, Kvitsau B, Buntzen S, Laurberg S, Krogh K. Long-term outcome and safety of transanal colonic irrigation for neurogenic bowel dysfunction. Spinal Cord 2009; 47: 545-549.

34 Christensen P, Kvitzau B, Krogh K, Buntzen S, Laurberg S. Neurogenic colorectal dysfunction - use of new antegrade and retrograde wash-out methods. Spinal Cord 2000; 38: 255-261.

35 Korsten MA, Fajardo NR, Rosman AS, Creasey GH, Spungen AM, Bauman WA. Difficulty with evacuation after spinal cord injury: colonic motility during sleep and effects of abdominal wall stimulation. JRRD 2004; 41: 95-99.

36 Hascakova-Bartova R, Dinant J-F, Parent A, Ventura M. Neuromuscular electrical stimulation of completely paralyzed abdominal muscles in spinal cord-injured patients: a pilot study. Spinal Cord 2008; 46: 445-450.

37 Lin VW, Nino-Murcia M, Frost F, Wolfe V, Hsiao I, Perkash I. Functional magnetic stimulation of the colon in persons with spinal cord injury. Arch Phys Med Rehabil 2001; 82: 167-173.

38 Lin VW, Kim KH, Hsiao I, Brown W. Functional magnetic stimulation facilitates gastric emptying. Arch Phys Med Rehabil 2002; 83: 806-810.

39 Mentes BB, Yuksel O, Aydin A, Tezcaner T, Leventoglu A, Aytac B. Posterior tibial nerve stimulation for faecal incontinence after partial spinal injury: preliminary report. Tech Coloproctol 2007; 11: 115-119. 
40 Tsai P-Y, Wang C-P, Chiu F-Y, Tsai Y-A, Chang Y-C, Chuang T-Y. Efficacy of functional magnectic stimulation in neurogenic bowel dysfunction after spinal cord injury. J Rehabil Med 2009; 41: 41-47.

41 Rajendran SK, Reiser JR, Bauman W, Zhang RL, Gordon SK, Korsten MA. Gastrointestinal transit after spinal cord injury: effect of cisapride. Am J Gastroenterol 1992; 87: 1614-1617.

42 Geders JM, Gaing A, Bauman WA, Korsten MA. The effect of cisapride on segmental colonic transit time in patients with spinal cord injury. Am J Gastroenterol 1995; 90: 285-289.

43 De Both PSM, de Groot GH, Slootman HR. Effects of cisapride on constipation in paraplegic patients: a placebo-controlled randomized double-blind cross-over study. Eur J Gastroenterol Hepatol 1992; 4: 1013-1017.

44 Krogh K, Jensen MB, Gandrup P, Laurberg S, Nilsson J, Kerstens R et al. Efficacy and tolerability of prucalopride in patients with constipation due to spinal cord injury. Scand J Gastroenterol 2002; 37: 431-436.

45 Segal JL, Milne N, Brunnemann SR, Lyons KP. Metoclopramideinduced normalization of impaired gastric emptying in spinal cord injury. Am J Gastroenterol 1987; 82: 1143-1148.

46 Korsten MA, Rosman AS, Ng A, Cavusoglu E, Spungen AM, Radulovic $\mathrm{M}$ et al. Infusion of neostigmine-glycopyrrolate for bowel evacuation in persons with spinal cord injury. Am J Gastroenterol 2005; 100: 1560-1565.

47 Rosman AS, Chaparala G, Monga A, Spungen AM, Bauman WA, Korsten MA. Intramuscular neostigmine and glycopyrrolate safely accelerated bowel evacuation in patients with spinal cord injury and defecatory disorders. Dig Dis Sci 2008; 53: 2710-2713.

48 Cardenas DD, Ditunno J, Graziani V, Jackson AB, Lammertse D, Potter $\mathrm{P}$ et al. Phase 2 trial of sustained-release fampridine in chronic spinal cord injury. Spinal Cord 2007; 45: 158-168.

49 Binnie NR, Creasey GH, Edmond P, Smith AN. The action of cisapride on the chronic constipation of paraplegia. Paraplegia 1988; 26: 151-158.

50 Longo WE, Woolsey RM, Vernava AM, Virgo KS, McKirgan L, Johnson FE. Cisapride for constipation in spinal cord injured patients: a preliminary report. J Spinal Cord Med 1995; 18: 240-244.

51 Binnie NR, Smith AN, Creasey GH, Edmond P. Constipation associated with chronic spinal cord injury: the effect of pelvic parasympathetic stimulation by the Brindley stimulator. Paraplegia 1991; 29: 463-469.

52 Chia YW, Lee TKY, Kour NW, Tung KH, Tan ES. Microchip implants on the anterior sacral roots in patients with spinal trauma: does it improve bowel function? Dis Colon Rectum 1996; 39: 690-694.

53 Kachourbos MJ, Creasey GH. Health promotion in motion: improving quality of life for persons with neurogenic bladder and bowel using assistive technology. Sci Nurs 2000; 17: 125-129.

54 MacDonagh RP, Sun WM, Smallwood R, Forster D, Read NW. Control of defecation in patients with spinal injuries by stimulation of sacral anterior nerve roots. BMJ 1990; 300: 1494-1497.

55 Gstaltner K, Rosen H, Hufgard J, Mark R, Scheri K. Sacral nerve stimulation as an option for the treatment of faecal incontinence in patients suffering from cauda equine syndrome. Spinal Cord 2008; 46: 644-647.

56 Johnston TE, Betz RR, Smith BT, Benda BJ, Mulcahey MJ, Davis R et al. Implantable FES system for upright mobility and bladder and bowel function for individuals with spinal cord injury. Spinal Cord 2005; 43: 713-723.

57 Holzer B, Rosen HR, Novi G, Ausch C, Nolbling N, Schiessel R. Sacral nerve stimulation for neurogenic faecal incontinence. $\mathrm{Br} \mathrm{J}$ Surg 2007; 94: 749-753.
58 Jarrett MED, Matzel KE, Christiansen J, Baeten CGMI, Rosen H, Bittorf B et al. Sacral nerve stimulation for faecal incontinence in patients with previous partial spinal injury including disc prolapse. Br J Surg 2005; 92: 734-739.

59 Valles M, Rodriguez A, Borau A, Mearin F. Effect of sacral anterior root stimulator on bowel dysfunction in patients with spinal cord injury. Dis Colon Rectum 2009; 52: 986-992.

60 Frisbie JH, Tun CG, Nguyen CH. Effect of enterostomy on quality of life in spinal cord injury patients. J Am Paraplegia Soc 1986; 9: 3-5.

61 Stone JM, Wolfe VA, Nino-Murcia M, Perkash I. Colostomy as treatment for complications of spinal cord injury. Arch Phys Med Rehabil 1990b; 71: 514-518.

62 Kelly SR, Shashidharan M, Borwell B, Tromans AM, Finnis D, Grundy DJ. The role of intestinal stoma in patients with spinal cord injury. Spinal Cord 1999; 37: 211-214.

63 Rosito O, Nino-Murcia M, Wolfe VA, Kiratli BJ, Perkash I. The effects of colostomy on the quality of life in patients with spinal cord injury: a retrospective analysis. J Spinal Cord Med 2002; 25: 174-183.

64 Branagan G, Tromans A, Finnis D. Effect of stoma formation on bowel care and quality of life in patients with spinal cord injury. Spinal Cord 2003; 41: 680-683.

65 Randell N, Lynch AC, Anthony A, Dobbs BR, Roake JA, Frizelle FA. Does a colostomy alter quality of life in patients with spinal cord injury? A controlled study. Spinal Cord 2001; 39: 279-282.

66 Luther SL, Nelson AL, Harrow JJ, Chen F, Goetz LL. A comparison of patient outcomes and quality of life in persons with neurogenic bowel: standard bowel care program vs colostomy. I Spinal Cord Med 2005; 28: 387-393.

67 Munck J, Simoens Ch, Thill V, Smets D, Debergh N, Fievet F et al. Intestinal stoma in patients with spinal cord injury: a restrospective study of 23 patients. Hepato-Gastroenterology 2008; 55: 2125-2129.

68 Safadi BY, Rosito O, Nino-Mursia M, Wolfe VA, Perkash I. Which stoma works better for colonic dysmotility in spinal cord injured patients? Am J Surg 2003; 186: 437-442.

69 Malone PS, Ransley PG, Kiely EM. Preliminary report: the antegrade continence enema. Lancet 1990; 336: 1217-1218.

70 Teichman JMH, Harris JM, Currie DM, Barber DB. Malone antegrade continence enema for adults with neurogenic bowel disease. J Urol 1998; 160: 1278-1281.

71 Teichman JMH, Zabihi N, Kraus SR, Harris JM, Barber DB. Longterm results for malone antegrade continence enema for adults with neurogenic bowel disease. Urology 2003; 61: 502-506.

72 Worsoe J, Christensen P, Krogh K, Buntzen S, Laurberg S. Long-term results of antegrade colonic enema in adult patients: assessment of functional results. Dis Colon Rectum 2008; 51: 1523-1528.

73 Ford MJ, Camilleri MJ, Hanson RB, Wiste JA, Joyner MJ. Hyperventilation, central autonomic control, and colonic tone in humans. Gut 1995; 37: 499-504.

74 Sloots CE, Felt-Bersma RJ, Meuwissen SG, Kuipers EJ. Influence of gender, parity, and caloric load on gastrorectal response in healthy subjects: a barstat study. Dig Dis Sci 2003; 48: 516-521.

75 Shafik A, El-Sibai O, Shafik IA. Physiologic basis of digital-rectal stimulation for bowel evacuation in patients with spinal cord injury: identification of an anorectal excitatory reflex. $J$ Spinal Cord Med 2000; 23: 270-275.

76 Furlan JC, Urbach DR, Fehlings MG. Optimal treatment for severe neurogenic bowel dysfunction after chronic spinal cord injury: a decision analysis. Br J Surg 2007; 94: 1139-1150.

77 Han TR, Kim JH, Kwon BS. Chronic gastrointestinal problems and bowel dysfunction in patients with spinal cord injury. Spinal Cord 1998; 36: 485-490. 